\title{
3D-Printed Microfluidic Sensor for Fluorescence Measurements
}

\author{
Witold Nawrot ${ }^{*}$, Wioletta Gzubicka1', Karol Malecha \\ ${ }^{1}$ Faculty of Microsystem Electronics and Photonics, Wrocław University of Science and Technology, 50-370 Wrocław, \\ 27 Wybrzeże Wyspiańskiego, Poland \\ * Corresponding author, e-mail: witold.nawrot@pwr.edu.pl
}

Received: 30 November 2019, Accepted: 07 July 2020, Published online: 11 November 2020

\begin{abstract}
In this work a fluorescent microfluidic sensor is developed, manufactured and characterized through additive manufacturing technique, using transparent photopolymer. A technology widely known as 3D printing is very suitable for tailor-made microfluidic chips, as it allows for rapid manufacturing. Optical structures can be developed using transparent materials, and this work describes a technique for increasing light transmission through such structure. A complete, inexpensive electronic device is described, containing microfluidic chip, light source, photodetector, microcontroller, communication interface and a multifunctional package with embedded connectors. Performed analyses show that performed surface modification significantly improves light transmission through structure, the material does not show autofluorescence, and whole device is suitable for fluorescence measurements.
\end{abstract}

\section{Keywords}

microsystem, optical sensor, transparent polymer, stereolithography (SLA), additive manufacturing

\section{Introduction}

Nowadays, the development and demand for new, better, more personalized and specialized devices has grown to a great extent. Traditional solutions require long processing time under supervision and generate significant equipment and personnel costs. Lab-on-chip solutions, composed commonly of microfluidic channels and inexpensive electronic components, seem to be an answer to those problems in some applications. Such small devices provide an ease of transport to places where access to the use of large equipment is difficult. Moreover, they can also be used as bedside devices. One of the tests that could be performed on biological samples in such microsystems is fluorescence measurement [1], which is utilized in presented device. The aim of this work is development, manufacturing and characterization of microfluidic sensor for determination of biological substances. The ultimate goal of our research is to develop portable and inexpensive Point of Care diagnostic devices based on microfluidic biosensors. Recent advances in Additive Manufacturing (AM), especially cost reduction of high resolution techniques, such as stereolithography and introduction of new materials, has made it very suitable for tailor made biochip manufacturing. AM is highly suitable for such applications, due to very short project to object time and high manufacturing flexibility - a series of various elements can be manufactured simultaneously.
Moreover, 3D printing is unparalleled in manufacturing costs. Both equipment and materials are inexpensive, there is no need for cleanroom environment. There is a variety of microfluidic sensors that are were applied for such purpose. The detection mechanism commonly relies either on electrochemical or optical phenomena. Electronic technologies are quite well developed, there are several that can incorporate microfluidic channels, such as semiconductor [2], ceramic [3] and polymer [4, 5]. On the other hand, optical microsystems require more complex manufacturing techniques [6]. The main issue is optical transparency- therefore silicon is commonly bonded with glass [7] and ceramics with PDMS (polydimethylsiloxane, an elastic silicon-based polymer [8]. Also, glass-PDMS systems are very popular due to reasonable manufacturing cost [9]. However, all of these technologies require specialized equipment and skilled staff. For example, in order to manufacture robust glass-PDMS or ceramic-PDMS structures, a bonding step is required. A plasma activation is most commonly used, which is a challenging process $[10,11]$. Therefore, although more capable, they are incomparably more expensive in low volume manufacturing and too complex for in-house manufacturing for many biomedical researches. This is a very important factor in microfluidic biochips, as most of them needs to be disposable. A more 
accessible approach is manufacturing PMMA microfluidic systems. Moradi et al. recently used this technique for development of a $\mathrm{pH}$ sensor [12]. They used a double sided tape for bonding consecutive layers. However, a more robust and long lasting solution is a mono-block 3D printed device. Additive manufacturing also allows for a high level integration of complex structures, such as valves or pumps, which can help to miniaturize the device and automate the measurement [13]. Several 3D printing methods had already been proven for microfluidic chip manufacturing [14-16]. A review by $\mathrm{Ni}$ et al. widely describes variety of 3D printed microfluidic devices, several of which have fluorescence detection, including PCR (Polymerase Chain Reaction) system and immunosensors [17]. In this work we focus on development of fluorescent, fully 3Dprinted sensor. The novelty in this work is a use of a very inexpensive stereolithography setup which can be incorporated at any laboratory. Moreover, a very simple modification step is described, which significantly increases transparency.

\section{Materials and methods}

Structures were manufactured using a Formlabs Form2 stereolithography (SLA) 3D printer. Stereolithography is a method of additive manufacturing, in which a mixture of mono- and oligomers is formed selectively into polymer by free radicals or cations, generated from photoinitiator under UV irradiation. The process is being carried out layer by layer, in order to form spatial structures.

As the working principle of described device is based on optical measurements, a transparent photocurable resin was used (Formlabs Clear). Therefore, characterization of the resin's optical parameters was necessary. The crucial parameter in optical measurements is light transmission of the microfluidic chip. Transparent SLA resins are available, however, surfaces are typically rough and significantly disperse light. Nevertheless, stereolithography is the most suitable AM technique for optically transparent object manufacturing, as it produces uniform structures. In comparison to other methods of 3D printing, consecutive layers cannot be distinguished in monoblock material. Therefore, the only obstacle for good transparency is surface finish. The most popular solution, not only in 3D printing, is polishing, which gives sufficient results. However, in our approach, a thin film of resin was deposited on the structure in order to fill in the unevenness and form a new outer layer. Due to surface tension of fluid, the new face is smooth enough to improve light transmission. Furthermore, as the layer is composed of the same material as bulk, there is no interface between them which would disperse light. The surface layer was deposited through dip coating, however, for more precise thickness control, a spin coating method can be used as well. In order to measure light transmission, structures with planar dimensions of $20 \mathrm{~mm} \times 20 \mathrm{~mm}$ and thickness of: $500 \mu \mathrm{m}, 750 \mu \mathrm{m}$, $1000 \mu \mathrm{m}$, and $1500 \mu \mathrm{m}$ were developed and then dip coated into transparent resin of which they were made of and then post-cured for between 5 to 30 minutes under a $9.1 \mathrm{~W} 405 \mathrm{~nm} \mathrm{UV}$ light in $60^{\circ} \mathrm{C}$. The timing of this process turned out to be crucial: it has to last long enough for the resin to fully cure but not so long that it starts to degrade and become yellowish; the optimal value seems to be 15 minutes. The whole procedure is illustrated in Fig. 1.

The transmittance of Formlabs Clear resin was assessed before and after such alteration. A StellarNet SilverNova 25 spectrophotometer $(190 \mathrm{~nm} \div 1110 \mathrm{~nm})$ was used for the transmittance measurements with a Thorlabs optical fibres $(180 \mathrm{~nm} \div 1200 \mathrm{~nm})$.

After the procedure of surface modification for improvement of light transmission was developed, a microfluidic chip designed and manufactured. It consists of two planar logarithmic spirals, one of which leads fluid to a center chamber and the other drains is out. This set of spirals is designed to provide mixing before measurement, in order to ensure uniform structure of analyte. The working

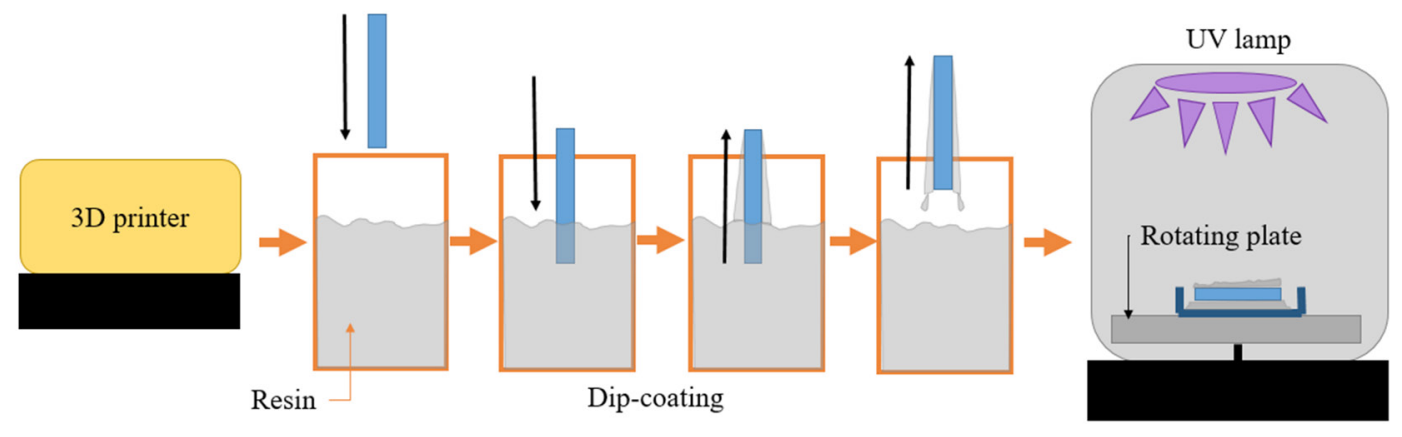

Fig. 1 Schematic of treatment microfluidic structure in modified process 
principle of such mixer is based on Dean vortices [18], as was previously described [8]. After printing, the channel was rinsed for 10 minutes in isopropyl alcohol using a peristaltic pump, in order to remove uncured resin. Then, the chip was dip coated in the same resin as it was made of and post-cured for 15 minutes under $9.1 \mathrm{~W} 405 \mathrm{~nm} \mathrm{UV}$ light in $60^{\circ} \mathrm{C}$. The view of the chip is shown in Fig. 2.

A peristaltic pump was used for inducing the fluid flow and a diode laser Osram PL450B with a peak wavelength at $460 \mathrm{~nm}$ and $80 \mathrm{~mW}$ maximum power was used for specimen excitation, as this wavelength corresponds with absorbance of fluorescein, which is widely used in fluorescence analyses. The package of transparent microfluidic chip was also 3D-printed, in order to insulate any incident light. Two SMA connectors were integrated in the package for easy optical fiber connection. Described system was developed in modular form, where light source is interchangeable and detection can be performed either by a compact colorimetric detector or by a spectrophotometer, however, development of very compact, integrated device can be easily achieved. The schematic of developed device is shown in Fig. 3.

After manufacturing and assembling, the whole device was characterized by fluorescence analyses, using fluorescein in concentrations of $0.1 \%$ and $0.01 \%$, diluted in ethanol. Before measurements, fluorescence analysis of pure ethanol was performed for a reference. The same measuring equipment was used as in transmittance measurements, described earlier.

\section{Results}

The measured values of the transmittance, depending on thickness of the structures are shown in Fig. 4. Modified structures are thicker than unmodified structures as they

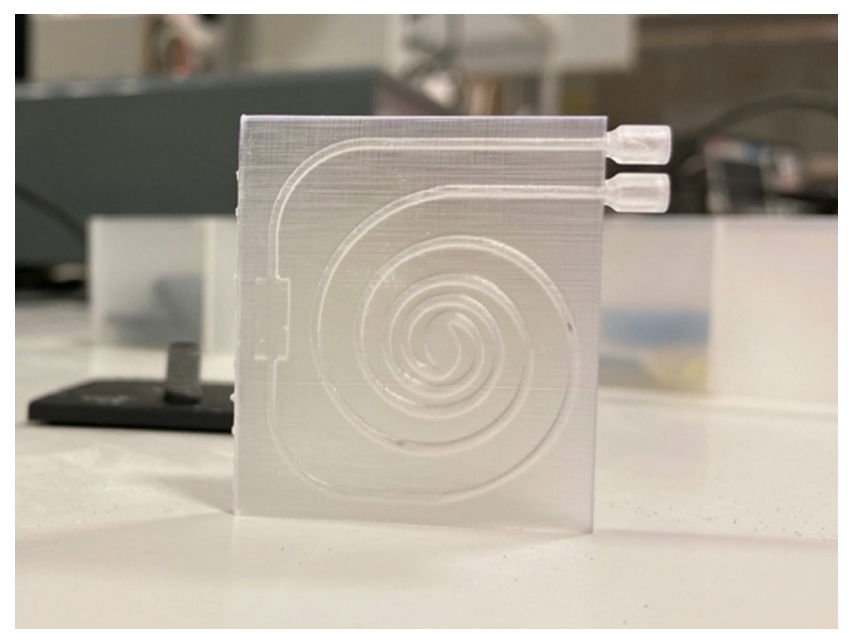

Fig. 2 A view of the microfluidic chip (before modification)

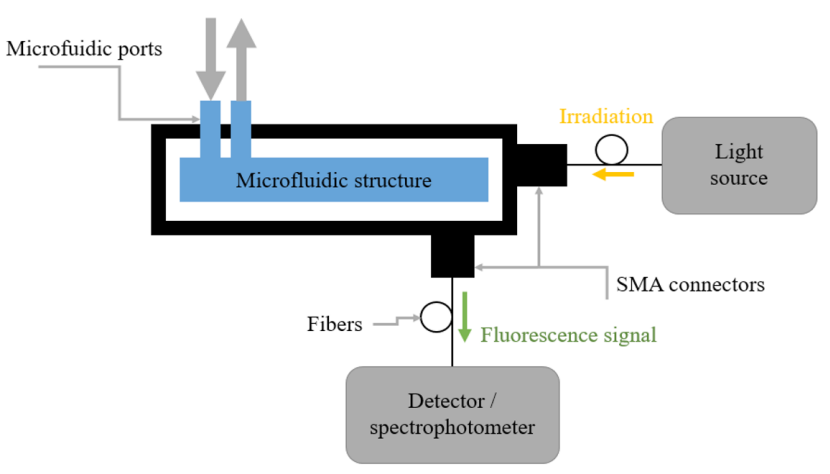

Fig. 3 Schematic of the sensor

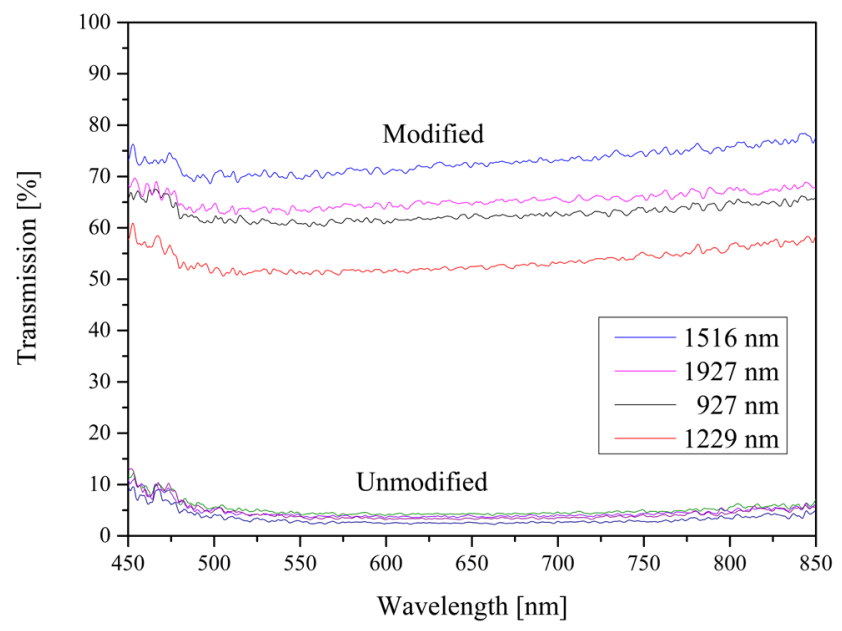

Fig. 4 Transmittance spectrum of Clear resin structures in dependence on their thickness

were dip-coated with an additional layer of liquid resin before curing, in order to obtain a smooth surface.

Nevertheless, despite the increased thickness, the modification significantly improves transmittance of the structures. Moreover, it can be observed that the quality of surface is more important than the thickness, proving that material itself does not have a significant attenuation and losses are mainly caused by the scattering on surface impurities. Obtained results are significantly better than for objects manufactured using Multi Jet Modelling method, where a photocurable material is selectively deposited and then the whole layer is cured by light irradiation. For VisiJet M3 Crystal, transmittance varies from $60 \%$ for $32 \mu \mathrm{m}$ thick structure to just $15 \%$ for $512 \mu \mathrm{m}$ thick structure [19]. A clear relation between transmission and object thickness can be observed in MJM, contrary to SLA technique presented in this work. The reason for that stereolithography produces a uniform material, whereas separate layers can be distinguished in the jet-printed objects.

In order to validate proper functioning of developed system, a series of fluorescence measurements were 
performed (Fig. 5). The excitation light can be clearly seen at $460 \mathrm{~nm}$ as well as wide spectra of flourescein response for two concentrations between $480 \mathrm{~nm}$ and $650 \mathrm{~nm}$. The signal from fluorescein is strong enough for the spectrophotometer to have a good resolution. In case of simple sensor measurement, a filter is needed in order to isolate fluorescein signal from excitation light. The reference measurement carries the most significant information, as no autofluorescence is observed, which is crucial for such measurement and is the main issue in many $3 \mathrm{D}$ printed materials, such as described earlier VisiJet M3 Crystal [19].

\section{Summary}

Performed research proves that it is possible to manufacture a reliable microfluidic sensor with optical detection, using stereolithography. This method eliminates a risk of spills or leaks outside the structure. All ports and connectors can be fully integrated, significantly reducing the assembly stage. Furthermore 3D printing methods provide rapid manufacturing are incomparably inexpensive in small volume manufacturing, making them ideal for tailor-made and disposable devices. Developed dip coating surface modification significantly improves transmittance, which is not highly dependent on wall thickness. Validation of the concept was performed by fluorescence measurements of fluorescein diluted in ethanol in different concentrations. Fluorescent signal is clearly visible and can be differed form $450 \mathrm{~nm}$ excitation laser. It proves that the microsystem topography and assembly is correct and can be used in chemical and

\section{References}

[1] Xu, D., Huang, X., Guo, J., Ma, X. "Automatic smartphone-based microfluidic biosensor system at the point of care", Biosensors and Bioelectronics, 110, pp. 78-88, 2018.

https://doi.org/10.1016/j.bios.2018.03.018

[2] Gallardo-Gonzalez, J., Baraket, A., Boudjaoui, S., Metzner, T., Hauser, F., Rößler, T., Krause, S., Zine, N., Streklas, A., Alcácer, A., Bausells, J., Errachid, A. "A fully integrated passive microfluidic Lab-on-a-Chip for real-time electrochemical detection of ammonium: Sewage applications", Science of The Total Environment, 653, pp. 1223-1230, 2019.

https://doi.org/10.1016/j.scitotenv.2018.11.002

[3] Horváth, E., Harsányi, G. "Optimization of fluidic microchannel manufacturing processes in low temperature co-fired ceramic substrates", Periodica Polytechnica Electrical Engineering (Archives), 54(1-2), pp. 79-86, 2010.

https://doi.org/10.3311/pp.ee.2010-1-2.08

[4] Chen, J., Zhou, Y., Wang, D., He, F., Rotello, V. M., Carter, K. R., Watkins, J. J., Nugen, S. R. "UV-nanoimprint lithography as a tool to develop flexible microfluidic devices for electrochemical detection", Lab on a Chip, 15(14), pp. 3086-3094, 2015.

https://doi.org/10.1039/C5LC00515A

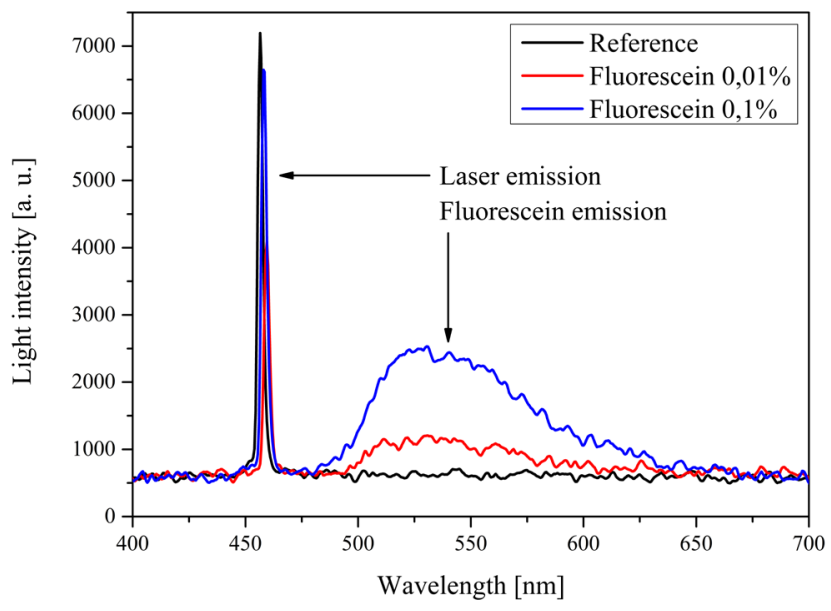

Fig. 5 Results of fluorescence measurements with excitation at $460 \mathrm{~nm}$

biological analyses. For example, the fluorescence measurements can be used for dopamine [20] or glucose [21] sensing. Crucially, Formlabs Clear does not show any autofluorescence. However, some reports show that this material may not be biocompatible [22], which is a common issue in $3 \mathrm{D}$ printed materials. Further research is needed in this area, including application of other available optically transparent resins or development of new composition. Another approach could be channel wall modification in the post processing stage, in order to provide chemically and biologically inert surface.

\section{Acknowledgement}

Research was funded by statutory funds of Wrocław University of Science and Technology.

[5] Fernández-la-Villa, A., Pozo-Ayuso, D. F., Castaño-Álvarez, M. "Microfluidics and electrochemistry: an emerging tandem for next-generation analytical microsystems", Current Opinion in Electrochemistry, 15, pp. 175-185, 2019.

https://doi.org/10.1016/j.coelec.2019.05.014

[6] Nawrot, W., Drzozga, K., Baluta, S., Cabaj, J., Malecha, K. "A Fluorescent Biosensors for Detection Vital Body Fluids' Agents", Sensors, 18(8), Article Number: 2357, 2018.

https://doi.org/10.3390/s18082357

[7] Bunge, F., van den Driesche, S., Waespy, M., Radtke, A., Belge, G., Kelm, S., Waite, A. M., Mirastschijski, U., Vellekoop, M. J. "Microfluidic oxygen sensor system as a tool to monitor the metabolism of mammalian cells", Sensors and Actuators B: Chemical, 289, pp. 24-31, 2019 https://doi.org/10.1016/j.snb.2019.03.041

[8] Nawrot, W., Fiedot-Toboła, M., Malecha, K. "PDMS - LTCC Lab on Chip for Photocatalytic Effect Analysis", In: 2017 21st European Microelectronics and Packaging Conference (EMPC) \& Exhibition, Warsaw, Poland, 2017, pp. 1-5. https://doi.org/10.23919/EMPC.2017.8346925 
[9] Gao, Y., Pan, X., Xu, S., Liu, Z., Wang, J., Yu, K., Wang, C., Yuan, H., Wu, S. "Fluorescence-enhanced microfluidic sensor for highly sensitive in-situ detection of copper ions in lubricating oil", Materials \& Design, 191, Article Number: 108693, 2020. https://doi.org/10.1016/j.matdes.2020.108693

[10] Hamidovic, M., Ender, F. "A Novel Method for Fabricating Microfluidic Devices Containing Immobilized Biological Specimens", Periodica Polytechnica Electrical Engineering and Computer Science, 63(2), pp. 85-93, 2019. https://doi.org/10.3311/PPee.13523

[11] Nawrot, W., Malecha, K. "Biomaterial Embedding Process for Ceramic-Polymer Microfluidic Sensors", Sensors, 20(6), Article Number: 1745, 2020. https://doi.org/10.3390/s20061745

[12] Moradi, V., Akbari, M., Wild, P. "A fluorescence-based pH sensor with microfluidic mixing and fiber optic detection for wide range $\mathrm{pH}$ measurements", Sensors and Actuators A: Physical, 297, Article Number: 111507, 2019. https://doi.org/10.1016/j.sna.2019.07.031

[13] Au, A. K., Bhattacharjee, N., Horowitz, L. F., Chang, T. C., Folch, A. "3D-printed microfluidic automation", Lab on a Chip, 15(8), pp. 1934-1941, 2015. https://doi.org/10.1039/c5lc00126a

[14] Ching, T., Li, Y., Karyappa, R., Ohno, A., Toh, Y. C., Hashimoto, M. "Fabrication of integrated microfluidic devices by direct ink writing (DIW) 3D printing", Sensors and Actuators B: Chemical, 297, Article Number: 126609, 2019. https://doi.org/10.1016/j.snb.2019.05.086

[15] Sochol, R. D., Sweet, E., Glick, C. C., Wu, S. Y., Yang, C., Restaino, M., Lin, L. "3D printed microfluidics and microelectronics", Microelectronic Engineering, 189, pp. 52-68, 2018. https://doi.org/10.1016/j.mee.2017.12.010
[16] Sharafeldin, M., Jones, A., Rusling, J. F. "3D-Printed Biosensor Arrays for Medical Diagnostics", Micromachines, 9(8), Article Number: 394, 2018. https://doi.org/10.3390/mi9080394

[17] Ni, Y., Ji, R., Long, K., Bu, T., Chen, K., Zhuang, S. "A review of 3D-printed sensors", Applied Spectroscopy Reviews, 52(7), pp. 623-652, 2017. https://doi.org/10.1080/05704928.2017.1287082

[18] Dean, W. R. "Note on the motion of a fluid in a curved pipe", The London, Edinburgh, and Dublin Philosophical Magazine and Journal of Science, 4(20), pp. 208-223, 1927. https://doi.org/10.1080/14786440708564324

[19] Walczak, R., Adamski, K., Pokrzywnicka, A., Kubicki, W. "Inkjet 3D Printing - Studies on Applicability for Lab-on-a-chip Technique", Procedia Engineering, 168, pp. 1362-1365, 2016. https://doi.org/10.1016/j.proeng.2016.11.377

[20] Baluta, S., Malecha, K., Zając, D., Sołoducho, J., Cabaj, J. "Dopamine sensing with fluorescence strategy based on low temperature co-fired ceramic technology modified with conducting polymers", Sensors and Actuators B: Chemical, 252, pp. 803-812, 2017. https://doi.org/10.1016/j.snb.2017.06.073

[21] Moschou, E. A., Sharma, B. V., Deo, S. K., Daunert, S. "Fluorescence Glucose Detection: Advances Toward the Ideal In Vivo Biosensor", Journal of Fluorescence, 14(5), pp. 535-547, 2004. https://doi.org/10.1023/B:JOFL.0000039341.64999.83

[22] Zhu, F., Friedrich, T., Nugegoda, D., Kaslin, J., Wlodkowic, D. "Assessment of the biocompatibility of three-dimensional-printed polymers using multispecies toxicity tests", Biomicrofluidics, 9(6), Article Number: 061103, 2015 https://doi.org/10.1063/1.4939031 